\title{
REVUE
}

\section{INTERNATIONA LE}

DE LA

\section{CROIX-ROUGE}

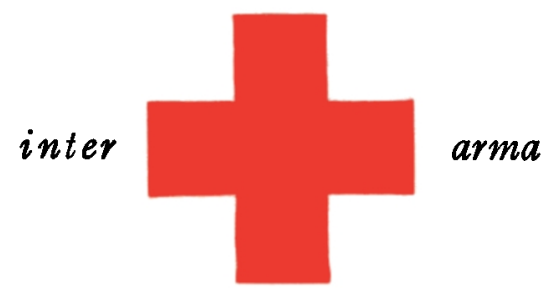

caritas 


\section{COMITÉ INTERNATIONAL DE LA CROIX-ROUGE}

\section{à Genève}

MM. MAX HUBER, docteur en droit, ancien président de la Cour permanente de justice internationale, président d'honneur (1923) 1

LÉOPOLD BOISSIER, docteur en droit, professeur honoraire de l'Université de Genève, ancien secrétaire général de l'Union interparlementaire, président (1946)

JACQUES CHENEVIERE, docteur ès lettres h. c. (1919)

M110 LUCIE ODIER, ex-chef du Service des infirmières-visiteuses de la Section genevoise de la CroixRouge suisse (1930)

MM. CARL J. BURCKHARDT, docteur en philosophie, ancien ministre de Suisse en France (1933)

MARTIN BODMER, docteur en philosophie h. c., vice-président (1940)

ERNEST GLOOR, docteur en médecine (1945)

PAUL CARRY, docteur en droit, professeur à l'Université de Genève (1946)

EDMOND GRASSET, docteur en médecine, professeur à l'Université de Genève, directeur de l'Institut d'hygiène (1946)

PAUL RUEGGER, ancien ministre de Suisse en Italie et en Grande-Bretagne, membre de la Cour Permanente d'Arbitrage (1948)

HENRI GUISAN, général, ancien commandant en chef de l'armée suisse (1948)

ALFREDO VANNOTTI, docteur en médecine, professeur à l'Université de Lausanne (1949)

RODOLFO OLGIATI, ancien directeur du Don suisse (1949)

M"to MARGUERITE VAN BERCHEM, ancien chef de service de l'Agence centrale des prisonniers de guerre (1951)

MM. FRÉDÉRIC SIORDET, avocat, conseiller du Comité international de la Croix-Rouge de 1943 à 1951, vice-président (1951)

MARCEL JUNOD, docteur en médecine, délégué du Comité international de la Croix-Rouge de 1935 a 1946 (1952)

GUILLAUME BORDIER, ingénieur dipl. E.P.F., M.B.A. Harvard, banquier (1955)

\section{Direction:}

MM. ROGER GALLOPIN, docteur en droit, directeur exécutif

JEAN S. PICTET, docteur en droit, directeur des Affaires générales

EDOUARD de BONDELI, sous-directeur, Services financiers et administratifs

CLAUDE PILLOUD, sous-directeur, Service juridique

1 Les années indiquées dans les parenthèses désignent les dates de nomination des membres du Comité international. 


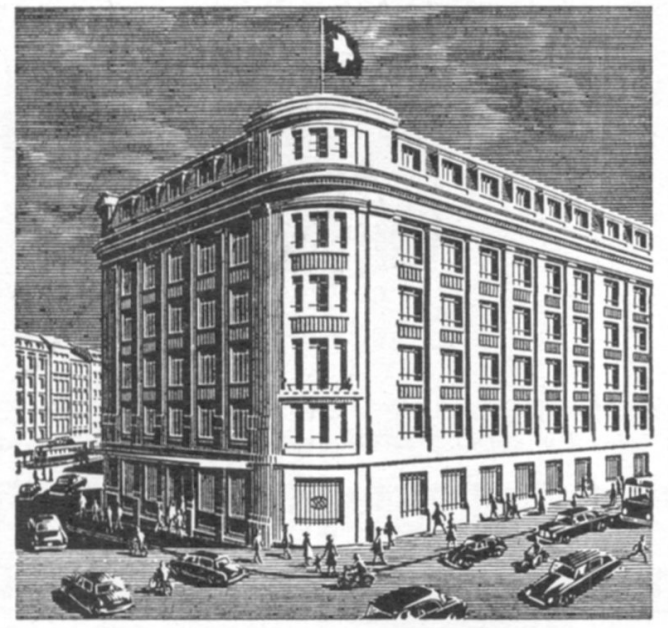

\section{SOCIETE DE}

\section{BANQUE SUISSE}

SCHWEIZERISCHER BANKVEREIN SOCIETÀ DI BANCA SVIZZERA SWISS BANK CORPORATION

\section{GENEVE}

BALE BIENNE LA CHAUX-DE-FONDS LAUSANNE NEUCHATEL ST.GALL SCHAFFHOUSE ZURICH LONDRES NEW-YORK

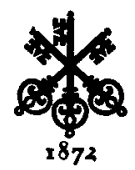

CAPITAL ET RÉSERVES FRS 275 MILLIONS 


\section{spécialités pharmaceutiques produits chimiques}

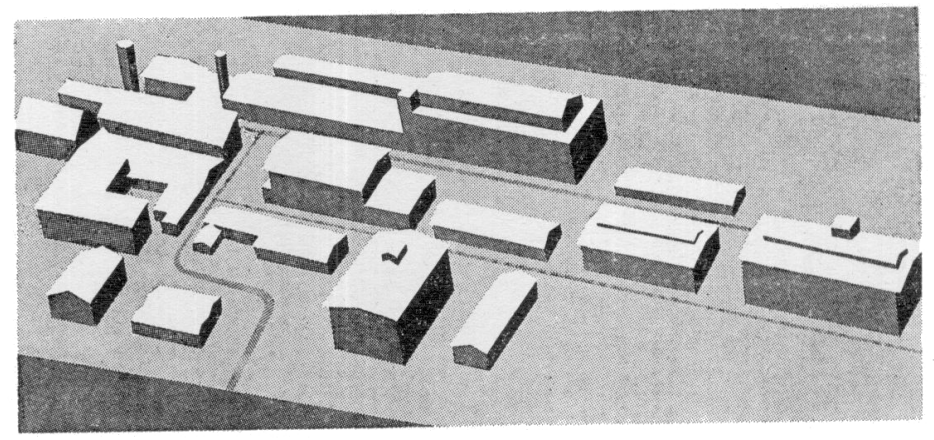

CILAG Société Anonyme Schaffhouse/Suisse

\section{SOCIÉTÉ ANONYME \\ FIDUCIAIRE SUISSE}

ORGANISATIONS • REVISIONS - EXPERTISES

QUESTIONS FISCALES 


\title{
REVUE INTERNATIONALE DE LA CROIX-ROUGE
}

\author{
TRENTE-NEUVIÈME ANNEE - No 460 \\ AVRIL 1957
}

$\begin{array}{lr}\text { SOMMAIRE } & \text { Pages } \\ \text { R. Boppe : Un des fondateurs de la Croix-Rouge : Louis Appia (II). . } \quad \text { I87 }\end{array}$

\section{COMITÉ INTERNATIONAL DE LA CROIX-ROUGE}

† Ernest Nobs, membre du CICR . . . . . . . . . . . . . $\quad \mathrm{I} 98$

† Heinrich Zangger, membre honoraire du CICR . . . . . . . 199

L'action du CICR en Hongrie . . . . . . . . . . . . . . . 200

Nouvelles brèves . . . . . . . . . . . . . . . 207

Mission du CICR dans la République fédérale allemande. . . . 215

Une mission du CICR au Kenya . . . . . . . . . . . . . . $2 \mathrm{I} 6$

Le délégué du CICR visite les camps d'internement de Chypre. . 216

Conventions de Genève . . . . . . . . . . . . . $2 \mathrm{I} 7$

Fonds de l'Impératrice Shôken (trente-sixième distribution des revenus)...................

Fondation en faveur du CICR: Situation et compte de résultats au 31 décembre 1956 . . . . . . . . . . . . . 22I

Fonds Augusta et Fonds de la Médaille Florence Nightingale: Situation au 31 décembre 1956 . . . . . . . . . . 224

\section{NOUVELLES DE SOCIÉTÉS NATIONALES}

Grande-Bretagne . . . . . . . . . . . 225

URSS.................. . . . 227

\section{CHRONIQUE}

Un épisode de la seconde guerre mondiale (W. Heudtlass) . . . 234

\section{BIBLIOGRAPHIE}




\section{SUPPLÉMENTS DE LA REVUE}

EN LANGUE ANGLAISE

The action of the ICRC in Hungary. - News Items. - An ICRC mission to the German Federal Republic. - A mission of the ICRC in Kenya. - The ICRC Delegate visits internment camps in Cyprus. - An episode of the Second World War (W. Heudtlass).

EN LANGUE ESPAGNOLE

LA acción del CICR en Hungría. - Noticias breves. - Misión del CICR en la República Federal de Alemania. - Misión del CICR en Kenia. - El delegado del CICR visita los campos de internamiento de Chipre. - Un episodio de la segunda guerra mundial (W. Heudtlass).

EN LANGUE ALLEMANDE

Die Aktion des IKRK in Ungarn. - Kurznachrichten. - Mission des IKRK in der Deutschen Bundesrepublik. - Eine Mission des IKRK in Kenya. - Der Delegierte des IKRK besucht die Internierungslager auf Zypern. - Ein Erlebnis aus dem zweiten Weltkrieg (W. Heudtlass).

LA

REVUE INTERNATIONALE DE LA CROIX-ROUGE est publiée, d la fin de chaque mois, par le Comité international de la Croix-Rouge

7, avenue de la Paix, Genève (Suisse) - Compte de chèques postaux I. 1767

Abonnement un an : Fr. 20,- ; le numéro Fr. 2,-

Pour les pays suivants : Algerie, Allemagne, Autriche, Belgique, Danemark, Finlande, France, Italie, Luxembourg, Maroc, Norvege, Pays-Bas, Portugal, Sarre, Suede, Tunisie, Vatican, on peut s'abonner aupres des bureaux de poste (Fr. s. 20,50).

Rédaction : JEAN-G. LOSSIER 